Canad. Math. Bull. Vol. 20 (1), 1977

\title{
ON A BIFURCATION THEOREM OF HOPF AND FRIEDRICHS*
}

\author{
H. I. FREEDMAN
}

\begin{abstract}
For the autonomous system $x^{\prime}=F(x, \varepsilon)$, the case where the second Hopf-Friedrichs condition fails is analyzed in that sufficient conditions for bifurcation of non-trivial periodic solutions to occur are given. An application to mathematical ecology is also discussed.
\end{abstract}

1. Introduction. Recently there has been much interest in the HopfFriedrichs bifurcation theorem. The theorem, first proved by Hopf [8] and later rediscovered by Friedrichs [7] in two dimensions, gives a set of sufficiency conditions under which an autonomous system with a scalar parameter may exhibit non-trivial, "small amplitude" periodic solutions for certain values of the parameter.

Various applications and extensions have been made of this theorem since the original work. Joseph and Sattinger [10] have applied the theorem to investigations of the Navier-Stokes equation, Pimbley [13] has utilized the theorem in developing and analyzing a model of immunity and Waltman [17] in a model of population growth. Alexander and Yorke [1] have considered global bifurcation, Chaffee [3] has considered a similar theorem for functional differential equations. Hsu [9], Poore [14] and others have given formulas for predicting the direction of bifurcation, whereas Takens [15] has considered the case when the parameter is itself a vector.

It is the main purpose of this paper to consider for the two dimensional system the case where one of the Hopf-Friedrichs conditions is violated, and to show that bifurcation may still occur. Results obtained will be similar to those obtained by Chaffee [2], who, in considering a similar theorem, showed that the critical point may bifurcate into several periodic solutions.

In the next section we will state our notation and techniques together with the preliminary analysis. In Section 4 we state and prove the main result. In the final section we apply the theorem to a perturbed system of equations which has been utilized in pest control theory.

Received by the editors October 8, 1976.

* Research for this paper was partically supported by the National Research Council of Canada, Grant No. NRC A-4823. This paper was partially written while on Sabbatical leave at the University of Minnesota, Minneapolis. 
The techniques used to prove the main theorem are similar to those used by Loud $[11,12]$. The calculations involved in this paper are long and tedious. Hence they will be eliminated from the text and only the results reported. Detailed calculations are available from the author upon request.

2. Preliminaries. We consider then the system

$$
x^{\prime}=F(x, \varepsilon) \quad\left('=\frac{d}{d t}\right)
$$

where $x, F$ are two-dimensional vectors and $\varepsilon$ is a real scalar parameter. We assume that for sufficiently small $\varepsilon$, there exists $a(\varepsilon)$ such that

$$
F(a(\varepsilon), \varepsilon)=0 \text {. }
$$

We further define the matrix $A(\varepsilon)$ by

$$
A(\varepsilon)=F_{x}(a(\varepsilon), \varepsilon),
$$

and for notational purposes let $a_{0}=a(0)$ and $A_{0}=A(0)$. Further let $F(x, \varepsilon)$ be analytic in a neighborhood of $\left(a_{0}, 0\right)$.

The Hopf-Friedrichs hypotheses (given in the Friedrichs formulation) which guarantee the bifurcation of periodic solutions either (a) for $\varepsilon>0$, or (b) for $\varepsilon>0$, or (c) all at $\varepsilon=0$, are

$$
\begin{aligned}
& \text { (H1) } \operatorname{tr} A_{0}=0, \quad \operatorname{det} A_{0}>0 \\
& \text { (H2) } \operatorname{tr} A_{\varepsilon}(0) \neq 0 .
\end{aligned}
$$

In the main theorem we will preserve $(\mathrm{H} 1)$, but violate $(\mathrm{H} 2)$.

At this point we introduce the notation which we shall use throughout the rest of this paper. We will need to discuss the matrices $A, A_{\varepsilon}$, and $A_{\varepsilon \varepsilon}$ at $\varepsilon=0$. Hence we set

$$
A_{0}=\left(\alpha_{i j}\right), \quad A_{\varepsilon}(0)=\left(\delta_{i j}\right), \quad A_{\varepsilon \varepsilon}(0)=\left(\theta_{i j}\right) .
$$

We will also need to consider higher order derivatives of $F$ in $x$ and $\varepsilon$ both in their entirety and componentwise. Hence by $F_{x x}(x, \varepsilon)$ we mean that tensor such that the $i$ th component of the vector $F_{x x} x^{2}$ is $\sum_{j, k}\left(\partial^{2} F_{i} / \partial x_{j} \partial x_{k}\right) x_{j} x_{k}$. We give similar meaning to $F_{x x x}$ and $F_{x x \varepsilon}$. Componentwise, we set

Then we let

$$
\begin{array}{ll}
\frac{\partial^{2} F_{i}\left(a_{0}, 0\right)}{\partial x_{1}^{p} \partial x_{2}^{q}}=\gamma_{p q}^{(i)}, & p+q=2, \\
\frac{\partial^{3} F_{i}\left(a_{0}, 0\right)}{\partial x_{1}^{p} \partial x_{2}^{q}}=\kappa_{p q}^{(i)}, & p+q=3 .
\end{array}
$$

$$
\omega=\sqrt{ }\left(\operatorname{det}\left|A_{0}\right|\right) .
$$

At this point we note that (H1), (H2) becomes (H1) $\alpha_{22}=-\alpha_{11}, \omega$ real, (H2) $\delta_{11}+\delta_{22} \neq 0$. We will need additional notations, but first we make the change of 
variables

$$
y=x-a(\varepsilon)
$$

Then $y$ satisfies the equation

$$
y^{\prime}=A(\varepsilon) y+f(y, \varepsilon) \quad\left('=\frac{d}{d t}\right)
$$

where

$$
f(y, \varepsilon)=F(a(\varepsilon)+y, \varepsilon)-A(\varepsilon) y .
$$

We note the following properties of $f$ which follow immediately from its definition.

$$
\begin{aligned}
f(0, \varepsilon) & =f_{\varepsilon^{n}}(0, \varepsilon)=0, & & n=1,2, \ldots, \\
f_{y}(0, \varepsilon) & =f_{\mathrm{y}^{n}}(0, \varepsilon)=0, & & n=1,2, \ldots, \\
f_{y^{n}}(0,0) & =F_{x^{n}}\left(a_{0}, 0\right), & & n=2,3, \ldots .
\end{aligned}
$$

We now let $y(t, \xi, \varepsilon)$ be that solution of system (2.9) such that $y_{1}(0, \xi, \varepsilon)=\xi$, $y_{2}(0, \xi, \varepsilon)=0$. We note an important property of $y(t, \xi, \varepsilon)$ :

$$
y(t, 0, \varepsilon)=\frac{d^{m} y_{\varepsilon^{n}}}{d t^{m}}(t, 0, \varepsilon)=0, \quad m, n=0,1, \ldots
$$

We now define the function $G(\tau, \xi, \varepsilon)$ by

$$
G(\tau, \xi, \varepsilon)=y(\tau, \xi, \varepsilon)-\left(\begin{array}{l}
\xi \\
0
\end{array}\right)
$$

and note that for arbitrary $\tau, \varepsilon$,

$$
G(\tau, 0, \varepsilon)=0
$$

by (2.14). Hence $\xi=0$ is always a solution of $G(\tau, \xi, \varepsilon)=0$, and we may define $H(\tau, \xi, \varepsilon)$ by

$$
H(\tau, \xi, \varepsilon)= \begin{cases}\xi^{-1} G(\tau, \xi, \varepsilon), & \xi \neq 0 \\ G_{\xi}(\tau, 0, \varepsilon), & \xi=0 .\end{cases}
$$

We clearly have $H\left(\tau_{0}, 0,0\right)=0$, where $\tau_{0}=2 \pi / \omega$.

In what follows, we will want the various partial derivatives of $H$ up to second order. First we note

$$
\begin{aligned}
H_{\tau} & =G_{\tau \xi}=y_{\xi}^{\prime} & & H_{\xi}=\frac{1}{2} G_{\xi \xi}=\frac{1}{2} y_{\xi \xi} \\
H_{\varepsilon} & =G_{\xi \varepsilon}=y_{\xi \varepsilon} & & H_{\tau \tau}=G_{\tau \tau \xi}=y_{\xi}^{\prime \prime} \\
H_{\tau \xi} & =\frac{1}{2} G_{\tau \xi \xi}=y_{\xi \xi}^{\prime} & & H_{\tau \varepsilon}=G_{\tau \xi \varepsilon}=y_{\xi \varepsilon}^{\prime} \\
H_{\xi \xi} & =\frac{1}{3} G_{\xi \xi \xi}=\frac{1}{3} y_{\xi \xi \xi \xi} & & H_{\xi \varepsilon}=\frac{1}{2} G_{\xi \xi \varepsilon}=\frac{1}{2} y_{\xi \xi \varepsilon} \\
H_{\varepsilon \varepsilon} & =G_{\xi \varepsilon \varepsilon}=y_{\xi \varepsilon \varepsilon}, & &
\end{aligned}
$$

where all functions are evaluated at $\left(\tau_{0}, 0,0\right)$. 
In order to define the last function we shall need, we will at this time compute $H_{\tau}, H_{\xi}$, and $H_{\varepsilon}$ explicitly. The techniques of computing these as well as the second partials later may be found in various papers of Loud, and in particular in $[11,12]$. It turns out that

$$
H_{\tau}\left(\tau_{0}, 0,0\right)=\left(\begin{array}{c}
\alpha_{11} \\
\alpha_{21}
\end{array}\right), \quad H_{\xi}\left(\tau_{0}, 0,0\right)=0
$$

$$
\begin{aligned}
& H_{\varepsilon}\left(\tau_{0}, 0,0\right) \\
& \qquad=\frac{\pi}{\omega^{3}}\left(\begin{array}{l}
-\left(2 \alpha_{11}^{2}+\alpha_{12} \alpha_{21}\right) \delta_{11}-\alpha_{11} \alpha_{21} \delta_{12}-\alpha_{11} \alpha_{12} \delta_{21}-\alpha_{12} \alpha_{21} \delta_{22} \\
-\alpha_{11} \alpha_{21} \delta_{11}-\alpha_{21}^{2} \delta_{12}-\alpha_{12} \alpha_{21} \delta_{21}+\alpha_{11} \alpha_{21} \delta_{22}
\end{array}\right) .
\end{aligned}
$$

Since $\operatorname{det} A_{0} \neq 0$, then $\alpha_{21} \neq 0$ and since $H\left(\tau_{0}, 0,0\right)=0$, by the implicit function theorem, it is possible to solve $H_{2}(\tau, \xi, \varepsilon)=0$ for $\tau(\xi, \varepsilon)$ near $\left(\tau_{0}, 0,0\right)$,

$$
\tau=\frac{2 \pi}{\omega}+\frac{\pi}{\alpha_{21} \omega^{3}}\left(\alpha_{11} \alpha_{21} \delta_{11}+\alpha_{21}^{2} \delta_{12}+\alpha_{12} \alpha_{21} \delta_{21}-\alpha_{11} \alpha_{21} \delta_{21}\right) \varepsilon+\text { H.O.T. }
$$

We then substitute for $\tau$ in $H_{1}(\tau, \xi, \varepsilon)$ and define

$$
J(\xi, \varepsilon)=H_{1}(\tau(\xi, \varepsilon), \xi, \varepsilon) .
$$

We note that

$$
J(0,0)=0
$$

and computing the first partial derivatives of $J$, we get

$$
I_{\xi}(0,0)=0, \quad J_{\varepsilon}(0,0)=\frac{\pi}{\omega} \operatorname{tr} A_{\varepsilon}(0) .
$$

3. The main theorem. Unless otherwise stated, throughout the rest of this paper, we shall assume that the condition $(\mathrm{H} 2)$ is violated, i.e.

$$
\delta_{22}=-\delta_{11} \text {. }
$$

This then implies by $(2.23)$ that $J_{\varepsilon}(0,0)=0$ and hence we will want to compute the second partial derivatives of $J(\xi, \varepsilon)$ at $(0,0)$. They are given in the obvious way by the second derivatives of $H$. These are computed from (2.18) by rather lengthy calculations utilizing Loud's techniques. The results are as follows:

$$
\begin{aligned}
J_{\xi \xi}(0,0)= & \frac{\pi}{4 \omega^{5}}\left[\left(-\alpha_{12} \alpha_{21} \kappa_{30}^{(1)}+2 \alpha_{11} \alpha_{21} \kappa_{21}^{(1)}+\alpha_{21}^{2} \kappa_{12}^{(1)}-\alpha_{12} \alpha_{21} \kappa_{21}^{(2)}\right.\right. \\
& \left.+2 \alpha_{11} \alpha_{21} \kappa_{12}^{(2)}+\alpha_{21}^{2} \kappa_{03}^{(2)}\right) \omega^{2}-\alpha_{11} \alpha_{12} \alpha_{21} \gamma_{20}^{(1) 2} \\
& +\alpha_{21}\left(2 \alpha_{11}^{2}-\alpha_{12} \alpha_{21}\right) \gamma_{20}^{(1)} \gamma_{11}^{(1)}+\alpha_{11} \alpha_{21}^{2} \gamma_{20}^{(1)} \gamma_{02}^{(1)}
\end{aligned}
$$


1977]

$$
\begin{aligned}
& -\alpha_{12}^{2} \alpha_{21} \gamma_{20}^{(1)} \gamma_{20}^{(2)}+\alpha_{11} \alpha_{12} \alpha_{21} \gamma_{20}^{(1)} \gamma_{11}^{(2)}+2 \alpha_{11} \alpha_{21}^{2} \gamma_{11}^{(1)} \\
& +\alpha_{21}^{3} \gamma_{11}^{(1)} \gamma_{02}^{(1)}+\alpha_{11} \alpha_{12} \alpha_{21} \gamma_{11}^{(1)} \gamma_{20}^{(2)}+\alpha_{11} \alpha_{21}^{2} \gamma_{11}^{(1)} \gamma_{02}^{(2)} \\
& +\alpha_{11} \alpha_{21}^{2} \gamma_{02}^{(1)} \gamma_{11}^{(2)}+\alpha_{21}^{3} \gamma_{02}^{(1)} \gamma_{02}^{(2)}-\alpha_{12}^{2} \alpha_{21} \gamma_{20}^{(2)} \gamma_{11}^{(2)} \\
& +\alpha_{11} \alpha_{12} \alpha_{21} \gamma_{20}^{(2)} \gamma_{02}^{(2)}+2 \alpha_{11} \alpha_{12} \alpha_{21} \gamma_{11}^{(2)} \\
& \left.-\alpha_{21}\left(2 \alpha_{11}^{2}-\alpha_{12} \alpha_{21}\right) \gamma_{11}^{(2)} \gamma_{02}^{(2)}-\alpha_{11} \alpha_{21}^{2} \gamma_{02}^{(2)}\right]
\end{aligned}
$$

$$
\begin{aligned}
J_{\xi \varepsilon}(0,0)= & \frac{\pi}{\omega^{3} \alpha_{21}}\left(2 \alpha_{11} \delta_{11}+\alpha_{21} \delta_{12}+\alpha_{12} \delta_{21}\right)\left(\alpha_{11} \gamma_{20}^{(2)}-\alpha_{21} \gamma_{20}^{(1)}\right) \\
J_{\varepsilon \varepsilon}(0,0)= & \frac{\pi}{\omega}\left(\theta_{11}+\theta_{22}\right)+\frac{\pi}{\omega^{3} \alpha_{21}}\left(7 \alpha_{11} \delta_{11}+4 \alpha_{21} \delta_{12}+4 \alpha_{12} \delta_{21}\right) \\
& \times\left(\alpha_{11} \delta_{21}-\alpha_{21} \delta_{11}\right) \\
& -\frac{4 \pi^{2}}{\omega^{2}}\left(2 \alpha_{11} \delta_{11}+\alpha_{21} \delta_{12}+\alpha_{12} \delta_{21}\right)^{2}
\end{aligned}
$$

We now state and prove the main theorem.

Theorem 3.1. Let $F(x, \varepsilon)$ be analytic in $x$, $\varepsilon$ in a neighborhood of $\left(a_{0}, 0\right)$. Let (H1) hold, but (H2) fail to hold. Further let

$$
J_{\xi \varepsilon}(0,0)^{2}-J_{\xi \xi}(0,0) J_{\varepsilon \varepsilon}(0,0)>0,
$$

where $J_{\xi \xi}(0,0), J_{\xi \varepsilon}(0,0), J_{\varepsilon \varepsilon}(0,0)$ are given by (3.2), (3.3), and (3.4) respectively. Then there is a bifurcation of non-trivial periodic solutions from $\varepsilon=0$.

Proof. By the definition of $y(t, \xi, \varepsilon)$, there will be "small amplitude" periodic solutions if we can find $\tau(\varepsilon), \xi(\varepsilon)$ such that $y(\tau(\varepsilon), \xi(\varepsilon), \varepsilon)=\left(\begin{array}{c}\xi(\varepsilon) \\ 0\end{array}\right)$ with $(\tau(\varepsilon), \xi(\varepsilon)) \rightarrow\left(\tau_{0}, 0\right)$ as $\varepsilon \rightarrow 0$. This will occur if we can solve $G(\tau, \xi, \varepsilon)=0$ for such $\tau$ and $\xi$ as functions of $\varepsilon$. Since we are interested in non-constant periodic solutions, this would be equivalent to solving $H(\tau, \xi, \varepsilon)=0$ for $\tau$ and $\xi$ as such functions of $\varepsilon$.

We have already solved $H_{2}$ for $\tau$ as a function of $\xi$ and $\varepsilon$ and substituted into $H_{1}$ to define $J(\xi, \varepsilon)$. The question then reduces to whether or not we can solve $J(\xi, \varepsilon)=0$ for $\xi$ as such a required function of $\varepsilon$. However, by the results contained in [4], we can so solve, given that $J$ and its first partial derivatives vanish at $(0,0)$, provided that $(3.5)$ holds. This completes the proof of the theorem.

Remark 3.2. Since in general the question of solving $J(\xi, \varepsilon)=0$ for $\xi$ as a function of $\varepsilon$ reduces to solving a quadratic equation for real solutions (guaranteed by (3.5)), there will be two distinct amplitudes $\xi$, both of order $\varepsilon$, giving the required periodic solutions. This corresponds to results obtained by Chaffee [2] for his system. Here, however, we may predict precisely when the 
two solutions reduce to one, namely when either $J_{\xi \xi}(0,0)=0$ or $J_{\xi \varepsilon}(0,0)=0$, or $J_{\varepsilon \varepsilon}(0,0)=0$.

Remark 3.3. Bifurcations of even more periodic solutions may occur if $J_{\xi \xi}(0,0)=J_{\xi \varepsilon}(0,0)=J_{\varepsilon \varepsilon}(0,0)=0$ or if one of the second partials is not zero, but $J_{\xi \varepsilon}-J_{\xi \xi} J_{\varepsilon \varepsilon}=0$. Then higher order critical cases of the implicit function theorem are involved.

Remark 3.4. In the case of the Hopf-Friedrichs bifurcation, i.e. $\operatorname{tr} A_{\varepsilon}(0) \neq 0$, then the condition

$$
J_{\xi \xi}(0,0) \neq 0
$$

is a sufficiency condition for the system at $\varepsilon=0$ not to be a center. This condition (together with the formula (3.2)) had been previously derived by Loud [12] provided that certain preliminary transformations had been made. It was also derived by Hsu [9] for higher dimensions (with preliminary transformations) as well as by others (e.g. Poore [14]).

4. An application. In this section we give an application to certain growth equations in mathematical ecology. Freedman and Waltman [6] considered the perturbed Lotka-Volterra system

$$
\begin{aligned}
& x^{\prime}=x(\alpha-\beta y)-\varepsilon f_{1}(x, y) \\
& y^{\prime}=y(-\gamma+\delta x)-\varepsilon f_{2}(x, y)
\end{aligned}
$$

in order to give sufficiency conditions for the existence of stable limit cycles. However, the case where

$$
\begin{array}{cl}
f_{i}\left(\frac{\gamma}{\delta}, \frac{\alpha}{\beta}\right)=0, & \sum_{i=1}^{2} f_{i x}^{2}\left(\frac{\gamma}{\delta}, \frac{\alpha}{\beta}\right)+f_{i y}^{2}\left(\frac{\gamma}{\delta}, \frac{\alpha}{\beta}\right) \neq 0, \quad i=1,2 \\
& f_{1 x}\left(\frac{\gamma}{\delta}, \frac{\alpha}{\beta}\right)+f_{2 y}\left(\frac{\gamma}{\delta}, \frac{\alpha}{\beta}\right)=0
\end{array}
$$

was not resolved.

Theorem 3.1 cannot be applied to system (4.1), since the system is of the form

$$
\begin{aligned}
& x^{\prime}=x F_{1}(y)-\varepsilon f_{1}(x, y) \\
& y^{\prime}=y F_{2}(x)-\varepsilon f_{2}(x, y)
\end{aligned}
$$

(discussed by Utz and Waltman [16] for $\varepsilon=0$ ) for which with (4.2) holding violates (3.5). However, an applicable generalization would be a system of the form

$$
\begin{aligned}
& x^{\prime}=x P(x, y)-\varepsilon f_{1}(x, y) \\
& y^{\prime}=y Q(x)-\varepsilon f_{2}(x, y),
\end{aligned}
$$

a special case of which was used in [5] in an analysis involving pest control by a natural enemy. 
We consider then system (4.4) under the assumptions that there exists $a>0$, $b>0$ such that

$$
P(a, b)=Q(a)=0, \quad P_{y}(a, b) Q_{x}(a)<0,
$$

and that (4.2) holds with $\gamma / \delta=a$ and $\alpha / \beta=b$. Then

$$
\begin{aligned}
A(0) & =\left(\alpha_{i j}\right)=\left(\begin{array}{cc}
0 & a P_{y} \\
b Q_{x} & 0
\end{array}\right) \\
A_{\varepsilon}(0) & =\left(\delta_{i j}\right)=\left(\begin{array}{cc}
-f_{1 x} & -f_{1 y} \\
-f_{2 x} & -f_{2 y}
\end{array}\right), \quad A_{\varepsilon \varepsilon}(0)=\left(\theta_{i j}\right)=0, \\
\gamma_{20}^{(1)} & =a P_{x x}, \quad \gamma_{11}^{(1)}=P_{y}+a P_{x y}, \quad \gamma_{02}^{(1)}=a P_{y y}, \quad \gamma_{20}^{(2)}=b Q_{x x} \\
\gamma_{11}^{(2)} & =Q_{x}, \quad \gamma_{02}^{2}=0, \quad \kappa_{30}^{(1)}=a P_{x x x}+3 P_{x x}, \quad \kappa_{21}^{(1)}=a P_{x x y}+2 P_{x y} \\
\kappa_{12}^{(1)} & =a P_{x y y}+P_{y y}, \quad \kappa_{03}^{(1)}=a P_{y y y}, \quad \kappa_{30}^{(2)}=b Q_{x x x}, \\
\kappa_{21}^{(2)} & =Q_{x x}, \quad \kappa_{12}^{(2)}=\kappa_{03}^{(2)}=0,
\end{aligned}
$$

where all functions are evaluated at $(a, b)$.

It is clear from (4.6) and (4.2) that $\operatorname{tr} A_{0}=\operatorname{tr} A_{\varepsilon}(0)=0$, det $A_{0}>0$. Hence by Theorem (3.1) there will be a bifurcation of periodic solutions from $\varepsilon=0$ provided (3.5) is satisfied, where here

$$
\begin{aligned}
J_{\xi \xi}(0,0)= & -\frac{\pi}{4 a^{3} b^{3} P_{y}^{4} Q_{x}^{4}}\left[a P_{y}\left(P_{x x x} Q_{x}-P_{x x} Q_{x x}\right)+2 P_{x x} P_{y}^{2} Q_{x}^{2}\right. \\
& \left.+b Q_{x}^{2}\left(P_{x y} P_{y y}-P_{x y y} P_{y}\right)\right] \\
J_{\xi \varepsilon}(0,0)= & -\frac{\pi P_{x x}}{a b P_{y}^{3} Q_{x}^{3}}\left(b Q_{x} f_{1 y}+a P_{y} f_{2 x}\right) \\
J_{\varepsilon \varepsilon}(0,0)= & \frac{4 \pi}{a^{3} b^{3} P_{y}^{3} Q_{x}^{3}}\left(b Q_{x} f_{1 y}+a P_{y} f_{2 y}\right) f_{1 x}-\frac{4 \pi^{2}}{a^{2} b^{2} P_{y}^{2} Q_{x}^{2}}\left(b Q_{x} f_{1 y}+a P_{y} f_{2 x}\right)^{2} .
\end{aligned}
$$

ACKNOWLedgements. I am deeply indebted to W. S. Loud for checking and verifying the calculations leading to formulas (3.2)-(3.4). I am also indebted to D. Sattinger, F. Takens, and P. Waltman for some helpful discussions during the writing of this paper.

\section{REFERENCES}

1. J. C. Alexander and J. A. Yorke, Global bifurcation of periodic orbits, Amer. J. Math. (to appear).

2. N. Chaffee, The bifurcation of one or more closed orbits from an equilibrium point of an autonomous differential system, J. Differential Equations 4 (1968), 661-679.

3. N. Chaffee, A bifurcation problem for a functional differential equation of finitely retarded type, J. Math. Anal. Appl. 35 (1971), 312-348.

4. H. I. Freedman, The implicit function theorem in the scalar case, Canad. Math. Bull. 12 (1969), 721-732.

5. H. I. Freedman, Graphical stability, enrichment, and pest control by a natural enemy, Math. Biosci, 31, (1976) 207-225.

6. H. I. Freedman and P. Waltman, Perturbation of two-dimensional, predator-prey equations, SIAM J. Appl. Math. 28 (1975), 1-10. 
7. K. O. Friedrichs, Advanced Ordinary Differential Equations, Gordon and Breach, 1965.

8. E. Hopf, Abzweigung einer periodischer Lösung von einer stationären Lösung eines Differentialsystems, Ber. Verh. Sächs. Akad. Wiss. Leipzig. Math-Nat. K1 95 (1943), 3-22.

9. I. D. Hsu, A higher order Hopf bifurcation formula and its application to Fitzhugh's nerve conduction equations, J. Math. Anal. Appl. (to appear).

10. D. D. Joseph and D. H. Sattinger, Bifurcating time periodic solutions and their stability, Arch. Rational Mech. Anal., 45 (1972), 79-109.

11. W. S. Loud, Periodic solutions of perturbed second-order autonomous equations, Mem. Amer. Math. Soc. \#47, 1964.

12. W. S. Loud, Behavior of the period of solutions of certain plane autonomous systems near centers, in "Contributions to Differential Equations, Vol. III", Wiley-Interscience, New York (1964), 21-36.

13. G. H. Pimbley, Jr., Periodic solutions of predator-prey equations simulating an immune response I, Math. Biosci. 20 (1974), 27-51.

14. A. B. Poore, On the theory and application of the Hopf-Friedrichs bifurcation theory, Arch. Rat. Mech. Anal. 60 (1976) 371-393.

15. F. Takens, Unfolding of certain singularities of vectorfields: generalized Hopf bifurcations, J. Differential Equations 14 (1973), 476-493.

16. W. R. Utz and P. E. Waltman, Periodicity and boundedness of solutions of generalized differential equations of growth, Bull. Math. Biophys. 25 (1963), 75-93.

17. P. E. Waltman, The equations of growth, Bull. Math. Biophys. 26 (1964), 39-43.

UNIVERSity of Alberta

UNIVERSITY OF MiNNESOTA 